\title{
THE
}

\section{Visualization of Multidimensional Clinical Data from Wearables on the Web and on Apps}

Travis M. Frink

University of Rhode Island

Joshua V. Gyllinsky

University of Rhode Island

Kunal Mankodiya

University of Rhode Island, kunalm@uri.edu

Follow this and additional works at: https://digitalcommons.uri.edu/ele_facpubs

The University of Rhode Island Faculty have made this article openly available.

Please let us know how Open Access to this research benefits you.

Terms of Use

This article is made available under the terms and conditions applicable towards Open Access

Policy Articles, as set forth in our Terms of Use.

\section{Citation/Publisher Attribution}

Frink, T. M., Gyllinsky, J. V., \& Mankodiya, K. (2017, November 3-5). Visualization of Multidimensional Clinical Data from Wearables on the Web and on Apps. 2017 IEEE MIT Undergraduate Research Technology Conference (URTC), Cambridge, MA. doi: 10.1109/URTC.2017.8284217

Available at: http://dx.doi.org/10.1109/URTC.2017.8284217

This Conference Proceeding is brought to you for free and open access by the Department of Electrical, Computer, and Biomedical Engineering at DigitalCommons@URI. It has been accepted for inclusion in Department of Electrical, Computer, and Biomedical Engineering Faculty Publications by an authorized administrator of DigitalCommons@URI.For more information, please contact digitalcommons-group@uri.edu. 


\section{Visualization of Multidimensional Clinical Data from Wearables on the Web and on Apps}

\author{
Travis M. Frink \\ Depts. of ECBE and CAS \\ University of Rhode Island \\ Kingston, United States \\ travis_frink@my.uri.edu
}

\author{
Joshua V. Gyllinsky \\ Depts. of ECBE, CSS \\ University of Rhode Island \\ Kingston, United States \\ jgyllinsky@my.uri.edu
}

\author{
Kunal Mankodiya \\ Dept. of ECBE \\ University of Rhode Island \\ Kingston, United States \\ kunalm@uri.edu
}

\begin{abstract}
Health related IoT is an important field and is changing health care by recording the critical amount of patient data necessary to identify patterns in patient data. This big data requires big data visualization to be useful to clinicians. We present the design architecture of a new visualization tool based on web and mobile platforms for use in the clinical setting. Implementation information of four diagram views of chronological patient data are discussed, representing both continuous calendars and days as pie charts.
\end{abstract}

Index Terms-Visualization, Big Data, Wearable IoT, Mobile, Electronic Health Record

\section{INTRODUCTION}

In recent years, devices for continuous data monitoring and logging of patients data have been technologies of growing interest in the medical field [11]. Advancements in smart textiles and wearables allow for vast amounts of data to be collected and made accessible to clinicians [9]. The large quantities of data associated with such systems are known as big data [5]. One of the benefits of big data for health care is that patterns can be recognized in a patient's lifestyle, allowing for better understanding and diagnosis of their medical issues [14]. Recording critical medical data in real time is especially useful in situations with immediate risks to health or life [6]. Luckily, along with advances in wireless and interconnecting technologies, allowing near real-time patient data to be transmitted and received over long distances, paradigms such as the Internet of Things or IoT have flourished [2]. Using sensor data, clinicians can receive constant input on their patient's health.

Big Data is defined by "4V's", volume, velocity, variety and value [5]. Data from medical IoT based real-time sensors qualify as Big Data due to the quantity of data generated (volume), the constant influx of data (velocity), time-dependent factors (variety) and its importance to patients health (value). Big data, can more easily be understood by computers than humans in database form. Due to these 4V's, Big Data is inherently complex, requiring advanced computational handling and methods for effective utilization. Graphing and other data visualization can make data more palatable, but for the big data collected from IoT environments, directly graphing the data is often insufficient. By using graphics and visuals, a complex system of data can be more easily described and understood by humans, with graphing going back at least as far as 1786 and health information in 1858 to document sanitary conditions of solders in 1858 [15]. A clinician may have several patients at any one point, each of which has devices recording data from several sensors. Measurements including heart rate, blood pressure and other inputs which require continuous and near continuous updating can be recorded to produce Big Data. Data visualization can allow detailed historical records to be analyzed without monopolizing the time-usage of the clinician and jeopardizing the personal attention required for other patients. In particular, this visualization method could be useful for heart rate measurements. Examining heart rate over intervals of several different durations has been shown to aid in assessing the health of a patient [10]. Data such as these have been used to help identify many types of health events including both heart attacks and pregnancy, and are thus clearly clinically useful [7].

Instead of displaying values directly, data visualization methods show numerically multidimensional data in the form of colors and shapes, providing a way to easily see several dimensions in a single display. The process of simplifying the dimensions of numerical data into a non-numerical form while not reducing the underlying data itself is known as dimensional reduction. Dimensional reduction occurs in the transformation of numerical data to a non-numerical form such as in data visualization. [12].

In our proposed solution, data is restructured for visualization on a temporal basis, such as by hour, day, week or month. Along these different periods, data is interpolated on longer time frames and displayed via visual markers such as shape and color. These colors and shapes can utilize familiar markers from everyday life, allowing a user to easily understand the meaning of a set of data without being explicitly told. Research related to this phenomenon dates back as far as 1982 and can be seen in both simple and complex tools such as maps and computers [8]. By reducing the dimensions of available data while displaying it in varying visual structures a user will be able to more fully understand it then in a case where dimensions are discrete and non-simplified [13]. Further, by using familiar markers the user can better determine the meaning of patterns in the data than in a typical graphical setting. 
Data of different types and classes can be placed in various visual relational and hierarchal structures. Using these aspects, the characteristics of a full data set can be displayed in a more compact setting while maintaining recognizable discrete dimensions. This can significantly decrease the interface area dedicated to data display while increasing the clarity of the data and its meaning to the user. Intuitive analysis of patient patterns can then be discovered through visual inspection by a clinician.

\section{Methods And Materials}

\section{A. Software Development Tools}

The proposed system utilizes a combination of smart phone and web browser based environments. Development was done using the IntelliJ based Android Studio by Google [1]. Google's Android SDK was the primary smart phone development tool, allowing the system to be used on Android based devices including smart phones and tablets.

The Android API provides a native Javascript interface which can then be injected into the web page. Custom defined functions built into these interfaces allow ease of data transfer and interaction between both Android and web page layers. The Javascript library D3.js (often shortened to just "D3") was the primary method of data input and visualization in web based development. Web pages were able to be displayed using a native Android web browser widget built into the application [3].

\section{B. Program Structure}

The program structure consists of 4 views, "Continuous Month", "Continuous Day", "Circular Day" and "MultiCircular Day" [figure 1]. Each view is contained in its own web-page and accessed from a native application [4]. Data were arranged into nested hierarchal data structures. These nests were structured temporally by date and time, with each time containing several types of labeled data for that period.

1) Continuous Month: A common tutorial on using D3 involves making some form of calendar and is usually oriented horizontally [4]. The primary interface of the application presents a continuous vertical calendar broken up by month and was inspired by horizontal calendar D3 tutorials [4a]. The current viewed year is displayed while the months and days are labeled accordingly on the calendar. Days are also marked by color, this is done by averaging data points of interest across each day and processing the average through a predetermined color gradient.

By selecting a day on the calendar the user is prompted with a web based dialog box, giving a basic summary of the day regarding predetermined important data. Double clicking a day opens another interface which displays the day in more detail by breaking it up into 24 hourly regions [4b].

2) Continuous Day: This display is also continuous and shows the corresponding date. Each hour displays averaged data represented by vertical bars varying in height. Bar colors are used to label the data. Each hour can also display multiple data points of interest by presenting multiple bars and labeling the axis according to the corresponding color.

3) Circular Day: From the same interface, another visual display of a particular days data is accessible via a pullup gesture from the bottom of the screen on the mobile application [4c].

A circular design is used for this interface, breaking the day into 24 radial sections representing the hours of the day. These sections are colored based on the underlying data, much like in the Continuous Month. Each hour can be selected to bring up another display table on the right. This table displays detailed data for the selected hour.

4) Multi-Circular Day: The user also has the option to stack these circular displays over the time span of several days, with each circle displaying data for a 24-hour interval [4d]. The size of these circular displays, as well as their adjacent distances, shrink as more are added. The radius of these circles decreases at a slower rate than the distances between the circles, causing the circles to overlap as more are added. These circular regions may then be rotated to reveal regions which are blocked by other circles. Distance between these circles $\Delta \mathrm{X}$ is calculated over a viewing window of width $\mathrm{W}$ and days to display $\mathrm{N}$ by

$$
\Delta X=C W / N
$$

where $\mathrm{C}$ is a scaling factor in $(0,1)$, decreasing $\Delta \mathrm{X}$ to allow overlapping as $\mathrm{C}$ decreases. The radius of each circle, $R$, is given by

$$
R=W \frac{(1-C(1-1 / N))}{2}
$$

allowing the circular regions to fit within the bounds of the viewing window.

\section{Alert System}

Beyond pure visual interpretation, there are some levels of automatic feedback available to the user. This is done using a threshold based system with predetermined user selected values for particular values of interest. When a threshold is passed, a visual notification will be displayed on the day the event occurred. If passed across multiple days than the notification will create a boxed selection around the affected days. The user is able to view these notifications to learn more about the events, such as the exact time and frequency associated with each event over different time periods.

\section{Example Use Case}

A clinician could use this application to monitor heart rate data over an extended time span with several patients. Heart rate may be recorded continuously over time, and at any moment the clinician may check the data from a mobile device to easily and immediately acquire each patients monitoring history. By doing this the clinician can get a general overview of each patient's physical activity per day across each week. The clinician can then select any day of interest to consider data on an hourly interval. If there are other sensors present 


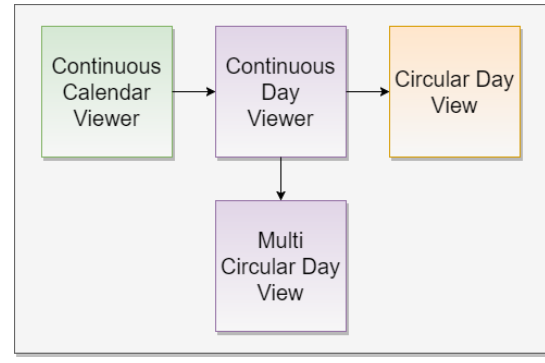

Fig. 1: Basic Program Structure

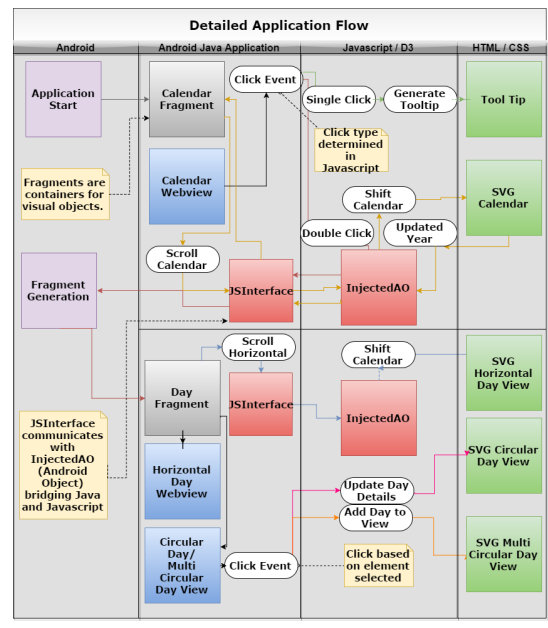

Fig. 2: Application Flow

they will also be able to view data recorded from these sensors. By further selecting the Multi-Circular Day View [4d] the clinician can compare data across several days. While seeing this view the clinician may be able to find events such as bed times as well as general patterns in the patient's weekly schedule.

With the ability to set alerts, the clinician can also find patterns based on predetermined points of interest. For example, the clinician may want to see when a patients heart rate is over $180 \mathrm{bpm}$. Through setting alerts, general patterns can be discovered on different time intervals. Furthermore, by examining each hour the clinician may note the exact point in time of the event, in this case a heart rate elevated beyond 180 bpm. This can be useful when a clinician needs to analyze the data leading up and following a point of interest in the data.

\section{RESULTS AND DISCUSSION}

\section{A. Choices and Reasoning}

The combination of both web and an native app granted the full capability of smart device's on-board sensors such as gyroscopes and magnetometers communicating through wireless connection systems such as Bluetooth, while minimizing common functions in graphing used in the display. Since the web portion can be accessed independently, it means any device which has web browser support is already minimally supported. This allows users of the application to find the same

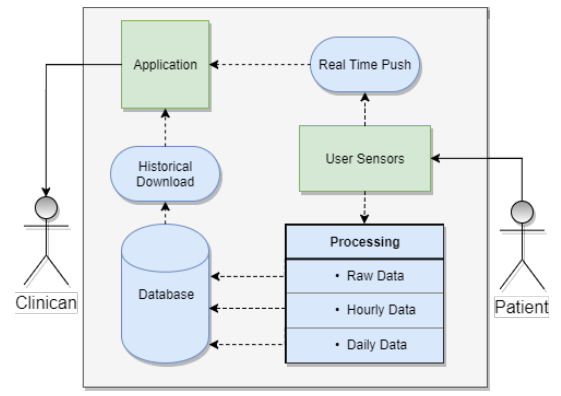

Fig. 3: Clincian and Patient Use Case

familiar interface available no matter where the application is accessed. Furthermore it minimizes development needed for supporting new platforms.

The application includes 3 primary levels of resolution. Data can be considered over months (long term), days (short term), and hours. The general flow of the program points in order of higher resolution Data across months is most easily seen in the initial Continuous Month [4a]. When the user goes to the next view they can then see data in detail across a single day or neighboring days through the Continuous Day [4b] or the Multi-Circular Day [4d]. These two views allow data comparison between multiple days or hours in a single setting. The last level of the resolution is the Circular Day, where the user can focus on hourly data for a single day, including detailed hourly summaries $[\mathbf{4 c}]$. Although these two views span time at a similar resolution, they do so while displaying data in a different manner. In the Continuous Day View, only one day is visible at a time, with the ability to easily access others by scrolling the web page. Data is concurrently displayed for each hour, showing how multiple dimensions of the data vary with time. The Multi-Circular Day View displays multiple days in a single screen with each day represented with 24 slices of a circle visible for each day through a color pallet. The concurrent use of both views allows the Continuous Day View to handle the comparison of multiple data sets while having the Multi-Circular Day View handle the comparison of a single data set at a time. Incorporating multiple sets of data in the Multi-Circular Day view would likely pollute the interface and cause confusion to the user, taking away from the simplicity of the design.

The alert system adds another factor to aid in visualization and understanding. Typically threshold based alert systems exist but are typically used in a stand-alone manner without significant visualization. Such methods do not simply show the user recurrence patterns in the data. Within the application, alerts are used to map out regions of data on the various resolutions present in each display. By having the alert system select regions at which a certain threshold of interest is achieved, the user may spot patterns and behaviors present in the data with greater ease. This alert system can notify the user about time periods of interest from weekly down to hourly intervals so that patterns in the data can be discovered across various resolutions. 


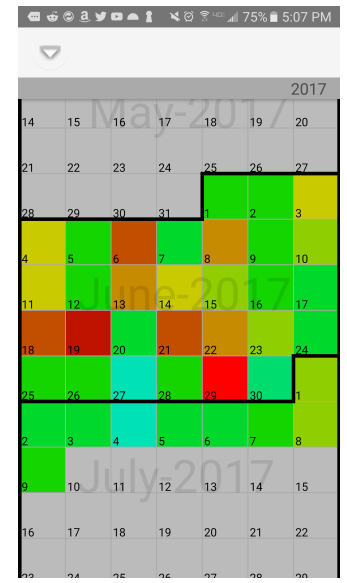

(a) Continuous Month

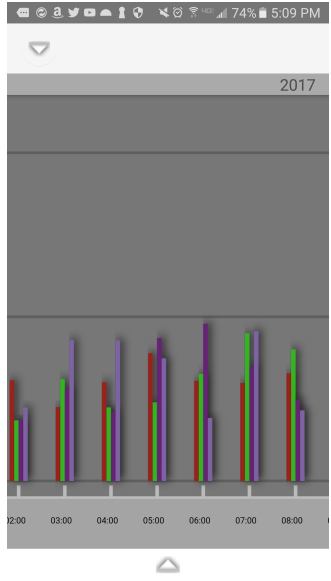

(b) Continuous Day

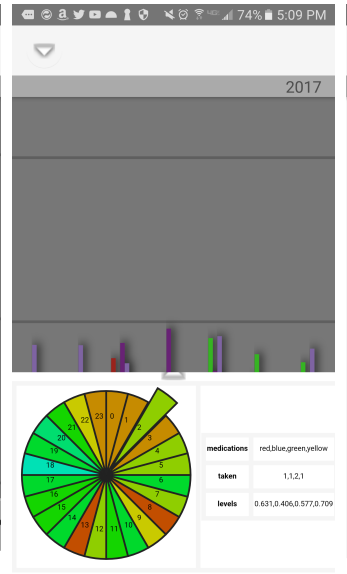

(c) Circular Day

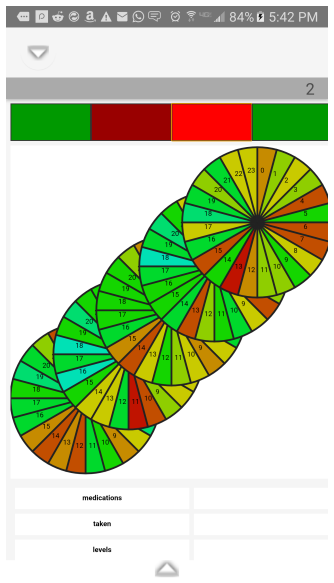

(d) Multi-Circular (In Web Browser)

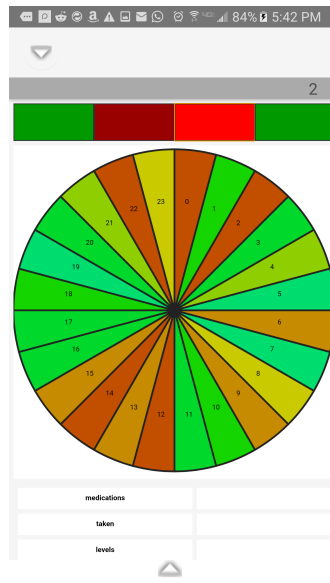

(e) Alternative Circular Day

Fig. 4: Graphing and visualizing example data. Showing features of visualizations in Android app implementation.

\section{CONCLUSiOn AND Future WORK}

Data visualization with integration into an IoT environment should allow real-time data to be better utilized by clinicians through visual inspection. With the proposed system clinicians may be better able to manage vast quantities of data generated from numerous patients. Most importantly, patient health can be strictly monitored while optimizing the clinicians time.

In the future several more features could be added to further aid usage and deployment of the application. Firstly, visualization is the primary objective in the near term, with additional views such as and improved polar area and sunburst diagrams. Data management is also an important aspect requiring further development. Storage solutions can be put into place which manage both real-time incoming data as well as long term archived data. A possible solution to such a problem includes taking the continuous received data and averaging it over the course of different time intervals such as hours, months, and weeks. This is similar to how the application manages data as it is input currently and would allow seamless transition to this type of database.

For further work and validation, the application could be used with sensors in real time to see its use and performance in a real-life setting to determine its effectiveness in various situations. Furthermore, clinical trials could be done with this application to see its performance in a real scenario, which is planned to be done in the future. During this period the clinicians would be able to record feedback based on their experience while using the application.

\section{ACKNOWLEDGMENT}

We are especially thankful for the D3 Library and the use of its examples provided by D3, as the Continuous Month View was largely inspired by and modified from examples provided by D3. It would be the authors' honor to merge some parts of it upstream eventually. Thank you to Alyssa Zisk and others who provided assistance with proof-reading and editing.

\section{REFERENCES}

[1] Android studio. https://developer.android.com/studio/index.html, 20002017.

[2] Debasis Bandyopadhyay and Jaydip Sen. Internet of things: Applications and challenges in technology and standardization. Wirel. Pers. Commun., 58(1):49-69, May 2011.

[3] M. Bostock, V. Ogievetsky, and J. Heer. $\mathrm{D}^{3}$; data-driven documents. IEEE Transactions on Visualization and Computer Graphics, 17(12):2301-2309, Dec 2011.

[4] Mike Bostock. Calendar view, 2017.

[5] Min Chen, Shiwen Mao, and Yunhao Liu. Big data: A survey. Mobile networks and applications, 19(2):171, 2014.

[6] T. Cibis, B. H. Groh, H. Gatermann, H. Leutheuser, and B. M. Eskofier. Wearable real-time ecg monitoring with emergency alert system for scuba diving. In 2015 37th Annual International Conference of the IEEE Engineering in Medicine and Biology Society (EMBC), pages 60746077, Aug 2015.

[7] James F Clapp. Maternal heart rate in pregnancy. American journal of obstetrics and gynecology, 152(6):659-660, 1985.

[8] Kathleen Hemenway. Psychological issues in the use of icons in command menus. In Proceedings of the 1982 Conference on Human Factors in Computing Systems, CHI '82, pages 20-23, New York, NY, USA, 1982. ACM.

[9] S. M. R. Islam, D. Kwak, M. H. Kabir, M. Hossain, and K. S. Kwak. The internet of things for health care: A comprehensive survey. IEEE Access, 3:678-708, 2015.

[10] Estela Kristal-Boneh, Mark Raifel, Paul Froom, and Joseph Ribak. Heart rate variability in health and disease. Scandinavian Journal of Work, Environment and Health, 21(2):85-95, 1995.

[11] T. Liang and Y. J. Yuan. Wearable medical monitoring systems based on wireless networks: A review. IEEE Sensors Journal, 16(23):8186-8199, Dec 2016.

[12] Laurens van der Maaten and Geoffrey Hinton. Visualizing data using t-sne. Journal of Machine Learning Research, 9(Nov):2579-2605, 2008.

[13] T. Mu, Y. J. Goulermas, and S. Ananiadou. Data visualization with structural control of global cohort and local data neighborhoods. IEEE Transactions on Pattern Analysis and Machine Intelligence, PP(99):1-1, 2017.

[14] Lauren Plant, Berly Noriega, Arjun Sonti, Nicholas Constant, and Kunal Mankodiya. Smart e-textile gloves for quantified measurements in movement disorders. In 2016 IEEE MIT Undergraduate Research Technology Conference, 2016.

[15] V. L. West, D. Borland, and W. E. Hammond. Innovative information visualization of electronic health record data: a systematic review. $J \mathrm{Am}$ Med Inform Assoc, 22(2):330-339, Mar 2015. 